\title{
Transition Restricted Gray Codes
}

\author{
Bette Bultena and Frank Ruskey \\ Department of Computer Science \\ University of Victoria \\ Victoria, B.C. V8W 3P6, Canada \\ abultena@csr.uvic.ca, fruskey@csr.uvic.ca
}

Submitted: November 16, 1995; Accepted: March 14, 1996.

\begin{abstract}
A Gray code is a Hamilton path $\mathrm{H}$ on the $n$-cube, $\mathrm{Q}_{n}$. By labeling each edge of $\mathrm{Q}_{n}$ with the dimension that changes between its incident vertices, a Gray code can be thought of as a sequence $H=t_{1}, t_{2}, \ldots, t_{N-1}$ (with $N=2^{n}$ and each $t_{i}$ satisfying $1 \leq t_{i} \leq n$ ). The sequence $H$ defines an (undirected) graph of transitions, $G_{H}$, whose vertex set is $\{1,2, \ldots, n\}$ and whose edge set $E\left(G_{H}\right)=\left\{\left[t_{i}, t_{i+1}\right] \mid 1 \leq i \leq N-1\right\}$. A $\mathrm{G}$-code is a Hamilton path $\mathrm{H}$ whose graph of transitions is a subgraph of $\mathrm{G}$; if $\mathrm{H}$ is a Hamilton cycle then it is a cyclic G-code. The classic binary reflected Gray code is a cyclic $\mathrm{K}_{1, \mathrm{n}}$-code. We prove that every tree $\mathrm{T}$ of diameter 4 has a $\mathrm{T}$-code, and that no tree $\mathrm{T}$ of diameter 3 has a $\mathrm{T}$-code.
\end{abstract}

Mathematical Reviews Subject Number: 05C45.

\section{Introduction}

The utility of the ubiquitous binary reflected Gray code is undisputed. See, for example, the books of Nijenhuis and Wilf [5], Reingold, Nievergelt, and Deo [6], and Wilf [8]. For certain applications, however, other Gray codes are desired. Many other Gray codes have been proposed, both for specific values of $n$ and general constructions. For example, Goddyn, Lawrence, and Nemeth [3], motivated by an issue in the design of photon detectors, study the problem of finding a Gray code that maximizes the minimum number of edges between the use of edges of the same dimension. In a recent paper, Savage and Winkler [7] find a Gray code in which all subsets of size $k$ appear before any of size $k+2$, and use this Gray code to improve the best known results on the notorious "middle levels" problem. 
The $n$-cube, $Q_{n}$, has vertex set, $V\left(Q_{n}\right)$, consisting of all bitstrings of length $n$. Its edge set, $E\left(Q_{n}\right)$, consists of all pairs, $[\mathbf{x}, \mathbf{y}]^{1}$, in which $\mathbf{x}$ and $\mathbf{y}$ have Hamming distance one; i.e., that differ in exactly one position. For $[\mathbf{x}, \mathbf{y}] \in \mathrm{E}\left(\mathrm{Q}_{\mathfrak{n}}\right)$, define $\tau(\mathbf{x}, \mathbf{y})$, the transition between $\mathbf{x}$ and $\mathbf{y}$, to be the position of the bit that is different. A Gray code is a Hamilton path $\mathrm{H}$ on the $\mathrm{n}$-cube, $\mathrm{Q}_{\mathfrak{n}}$; a cyclic Gray code is a Hamilton cycle on $\mathrm{Q}_{\mathfrak{n}}$. Given a Gray code $\mathrm{H}=\mathbf{b}_{1}, \mathbf{b}_{2}, \ldots, \mathbf{b}_{N}$, where $N=2^{n}$, its transition sequence, $\tau(H)$, is the sequence $\tau(H)=t_{1}, t_{2}, \ldots, t_{N-1}$, where $t_{i}=\tau\left(b_{i}, b_{i+1}\right)$. If $H$ is a cyclic Gray code, then in a cyclic transition sequence we append the additional transition $t_{N}=\tau\left(\mathbf{b}_{N}, b_{1}\right)$. The transitions $t_{i-1}$ and $t_{i}$ are said to delimit the bitstring $b_{i}$. The sequence $\tau(H)$ defines an (undirected) graph of transitions, $\mathrm{G}_{\mathrm{H}}$, whose vertex set is $[n]=\{1,2, \ldots, n\}$ and whose edge set is $E\left(G_{H}\right)=\left\{\left[t_{i}, t_{i+1}\right] \mid 1 \leq i \leq N-1\right\}$.

A motivation for this paper was the construction of a particular type of Hamilton cycle in the cube-connected-cycle, $\mathrm{CCC}_{n}$. A cube-connected-cycle is a certain cubic Cayley graph of the wreath product of an n-cycle and an n-cube, and is a well-known topology for computer networks. Formally, the vertex set $V\left(C C_{n}\right)$ consists of all pairs $(d ; x)$ where $\mathbf{x}$ is a length $n$ bitstring and $d \in \mathbb{Z}_{n}$ is an integer mod $n$. The edge set $E\left(C C C_{n}\right)$ is as shown below.

$$
\begin{aligned}
E\left(\operatorname{CCC}_{\mathfrak{n}}\right)= & \left\{[(\mathrm{d} ; \mathbf{x}),(\mathrm{d}+1 ; \mathbf{x})] \mid x \in \mathrm{V}\left(\mathrm{Q}_{\mathfrak{n}}\right) \text { and } 0 \leq \mathrm{d}<\mathrm{n}\right\} \quad \cup \\
& \left\{[(\mathrm{d} ; \mathbf{x}),(\mathrm{d} ; \mathbf{y})] \mid[\mathbf{x}, \mathbf{y}] \in \mathrm{E}\left(\mathrm{Q}_{\mathfrak{n}}\right) \text { and } 0 \leq \mathrm{d}<\mathrm{n}\right\}
\end{aligned}
$$

A basic fact about cube-connected-cycles is that they are Hamiltonian; an easily understood proof may be found Leighton [4]. We would like to find a particular type of Hamilton cycle - one that contiguously traverses all vertices of the form $(\mathrm{d} ; \mathbf{x})$, for $\mathbf{x}$ fixed, before moving onto a vertex with a different value of $\mathbf{x}$. Such a Hamilton cycle can be written in the form $\kappa_{1}, \kappa_{2}, \ldots, \kappa_{N}$, with $N=2^{n}$, where each $\kappa_{i}$ has the form (arithmetic done mod $n$ )

$$
\begin{aligned}
& \left(d_{i} ; x_{i}\right),\left(d_{i}+1 ; x_{i}\right),\left(d_{i}+2 ; x_{i}\right), \ldots,\left(d_{i}+(n-1) ; x_{i}\right), \text { or } \\
& \left(d_{i} ; x_{i}\right),\left(d_{i}-1 ; x_{i}\right),\left(d_{i}-2 ; x_{i}\right), \ldots,\left(d_{i}-(n-1) ; x_{i}\right) .
\end{aligned}
$$

The sequence $x_{1}, x_{2}, \ldots, x_{N}$ must be a Hamilton cycle in $Q_{n}$, and $d_{1}, d_{2}, \ldots, d_{N}$ is its transition sequence. Clearly, we must have $d_{i}=d_{i-1} \pm 1$.

The question naturally occurs as to whether a Hamilton cycle $\mathrm{H}$ in the $\mathrm{n}$-cube can be "lifted" to a Hamilton cycle in the cube-connected cycle $\mathrm{CCC}_{n}$ by always traversing sucessively all vertices of an $n$-cycle, either in increasing or decreasing order, as described above. Such a Hamilton cycle exists if and only if the dimension of the successive edges used in $\mathrm{H}$ differs by one $(\bmod n)$. This question is a special case of the problems considered below.

\footnotetext{
${ }^{1}$ Lower case bold letters always denote bitstrings.
} 
Given any graph $\mathrm{G}$, with $n$ vertices, a $\mathrm{G}$-code is a Hamilton path on $\mathrm{Q}_{\mathfrak{n}}$ whose graph of transitions is a subgraph of G. A cyclic G-code is a Hamilton cycle whose graph of transitions is a subgraph of $\mathrm{G}$. Every G-code has a unique G-transition sequence, whose length is $2^{\mathrm{N}}$ if the G-code is cyclic, and is $2^{\mathrm{N}}-1$ otherwise. In either case, we refer to the starting vertex and ending vertex as those numbered 1 and $2^{\mathrm{N}}-1$ in the sequence. Note that to prove the existence of a G-code for graph G, it is sufficient to produce a G-transition sequence. The question raised in the previous paragraph amounts to that of finding a cyclic $C_{n}$-code, where $C_{n}$ denotes an n-cycle.

The classic binary reflected Gray code (BRGC) has transition sequence $T_{n}$ defined recursively as follows. The initial condition is $T_{0}=1$ and if $n>0$, then $T_{n}=T_{n-1}, n, T_{n-1}$. For example, $T_{4}=1,2,1,3,1,2,1,4,1,2,1,3,1,2,1$. The cyclic transition sequence for the BRGC is $T_{n}, n$. For the BRGC the graph of transitions is $K_{1, n-1}$. The graph $K_{1, n-1}$ for $n \geq 3$ is called a star, denoted $S_{n}$. It is a tree with one central vertex and $n-1$ leaves.

The following useful theorem is due to Gilbert [2].

Theorem 1.1 The following statements characterize non-cyclic and cyclic transition sequences:

- Let $\mathrm{T}$ be a sequence of $2^{\mathrm{n}}-1$ integers from $[\mathrm{n}]$. The sequence $\mathrm{T}$ is a transition sequence if and only if every non-empty consecutive subsequence of $\mathrm{T}$ contains some integer an odd number of times.

- Let $\mathrm{T}$ be a sequence of $2^{\mathrm{n}}$ integers from $[\mathrm{n}]$. The sequence $\mathrm{T}$ is a cyclic transition sequence if and only if every non-trivial consecutive subsequence of $\mathrm{T}$ contains some integer an odd number of times, and every integer in [n] occurs in $\mathrm{T}$ an even number of times.

We will also think in an algebraic sense of transitions as operations on bitstrings, so that bt denotes the bitstring that results from flipping the $t^{\text {th }}$ bit of $\mathbf{b}$. We note the following obvious equalities. The operation is commutative: bst $=$ bts. The operation is an involution: $\mathbf{b t t}=\mathbf{b}$ or, equivalently, if $\mathbf{b t}=\mathbf{c}$, then $\mathbf{c t}=\mathbf{b}$.

A graph $\mathrm{G}$ is completely Gray if there is a G-transition sequence starting from every vertex of $G$. Observe that if there is a cyclic G-code, then $G$ is completely Gray. A graph $\mathrm{G}$ is Gray-connected if, for every pair of vertices $u$ and $v$, there is a G-transition sequence starting at $u$ and ending at $v$. A bipartite graph $G$ is Gray-laceable if, for every pair of vertices $u$ and $v$ in the same partite set, there is a G-transition sequence starting at $u$ and ending at $v$.

The diameter of a graph $\mathrm{G}$ is the maximum distance between any two vertices of $\mathrm{G}$. For a tree $\mathrm{T}$ the diameter is thus the length of the longest path in $\mathrm{T}$. A center of a tree 
THE ELECTRONIC JOURNAL OF COMBINATORICS 3 (1996), \#R11

is a vertex of minimum distance from all others. Every tree has one or two centers, and if it has two, then they are adjacent. A tree with one center is unicentral, a tree with two is bicentral.

We will refer to several standard graphs. The complete graph is denoted $K_{n}$. The complete bipartite graph is denoted $K_{n, m}$. The path with $n$ vertices (and $n-1$ edges) is denoted $P_{n}$. The cycle of length $n$ is denoted $C_{n}$.

\section{Undirected G-codes}

Below are two simple lemmata.

Lemma 2.1 If there is a G-code, then $\mathrm{G}$ is connected.

Lemma 2.2 If there is a G-transition sequence starting at $u$, then it must end at a vertex $v$ of $\mathrm{G}$ for which there is an even length path from $u$ to $v$. In particular, if $\mathrm{G}$ is bipartite, then a G-transition sequence must start and end at vertices in the same partite set.

Proofs: Every bit must change at least once in a G-code. This means that each vertex is encountered at least once in the G-transition sequence. Therefore, $G$ must be connected.

If a G-transition sequence starts at $u$, then it lists $2^{\mathfrak{n}}-2$ other vertices, which is an even number. By the property of bipartite graphs, the last vertex is in the same partite set as $u$.

Stars have interesting properties and are useful as subgraphs in succeeding constructions.

Lemma 2.3 (a) There is a cyclic $\mathrm{S}_{\mathrm{n}}$-transition sequence which starts and ends at the central vertex. (b) For any leaves $u$ and $v$ of $S_{n}$, there is a cyclic $S_{n}$-transition sequence which starts at $u$ and ends at $v$ if and only if $u \neq v$.

Proof: (a) Note that the BRGC's graph of transitions is $S_{n}$, and that $T_{n}$ begins and ends on the central vertex 1 .

(b) To show the necessity of $u \neq v$, let $\Sigma$ be the $S_{n}$-transition sequence that starts at $u$ and ends at $v$. Let $r$ be the central vertex. We can create a cyclic transition sequence, $\Sigma_{c}=t_{1}, t_{2}, \ldots, t_{N}$, with $u=t_{1}, v=t_{N-1}$, and $r=t_{N}$, where $N=2^{n}$, that meets the requirements of Theorem 1.1. Because $\Sigma_{c}$ is cyclic, we can start on $t_{N-1}$, producing a subsequence $t_{N-1}, t_{N}, t_{1}, t_{2}=v$ rur, which is illegal when $u=v$.

Consider $S_{n}$, with distinct leaves $u$ and $v$, and central vertex, $r$. Relabel $r$ as $v$ and remove the leaf $v$, creating $S_{\mathfrak{n}-1}$. Using the BRGC and starting at $u$, we can form a cyclic 


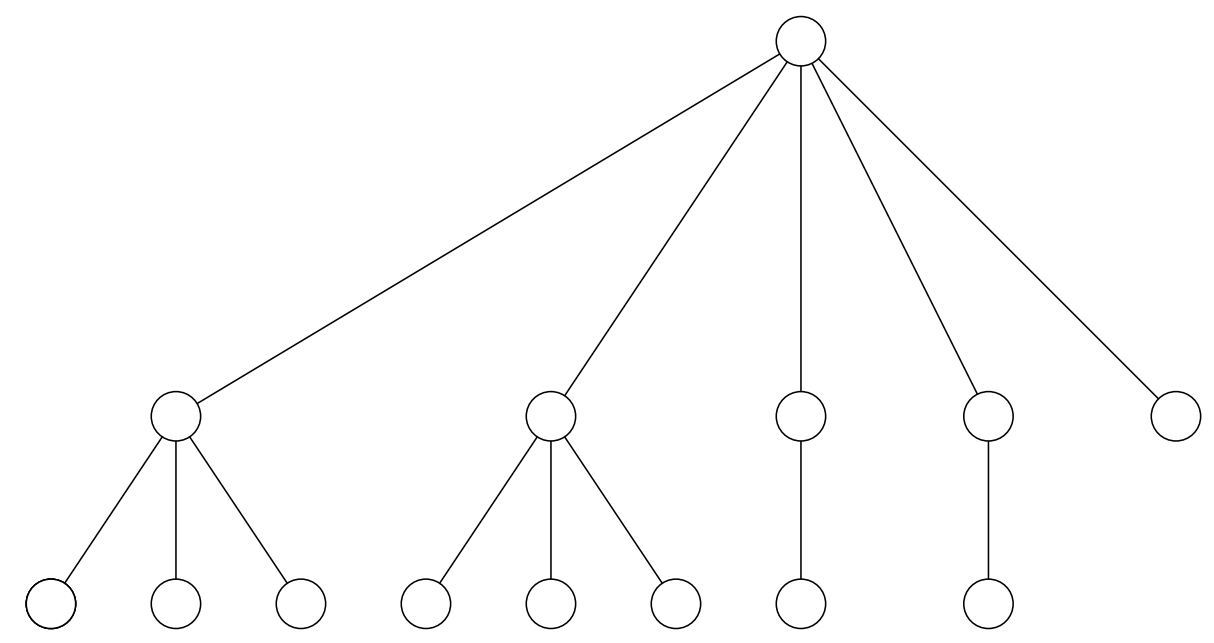

Figure 1: The short tree $T_{5}(3,3,1,1,0)$.

transition sequence, $\Sigma_{c}=u v \cdots v p v$, where $p$ is any leaf in $S_{\mathfrak{n}-1}$, and the length of the sequence is $2^{\mathfrak{n}-1}$. We can then place an $r$ between each $t_{i}, t_{i+1}$ pair, $\left(1 \leq i \leq 2^{n-1}-1\right)$, producing a new sequence $\Sigma=$ urvr $\cdots$ rvrprv, with length $2^{\mathfrak{n}}-1$. Note that any proper subsequence of the original sequence contains some vertex an odd number of times. Adding the r's maintains this property, and by appending an $r$ to the end of $\Sigma$, we create the condition that every vertex is visited an even number of times. This means that we can create a cyclic transition sequence from $\Sigma$. The graph of transitions for $\Sigma$ is clearly $S_{n}$ and the starting and ending leaves have been chosen arbitrarily.

Corollary 2.1 The complete graph $\mathrm{K}_{\mathrm{n}}$ is Gray-connected.

Proof: Note that $K_{n}$ contains $S_{n}$ as a spanning tree. Because you can choose any vertex as the root or any two distinct vertices as leaves, all combinations of start and finish vertices can produce a G-transition sequence.

Define a short tree to be a rooted tree of height at most 2, where the height of a tree is the length of the longest path from the root to a leaf. A short tree may be specified by the number of children of each of the nodes at level 1 . Let $T_{t}\left(n_{1}, n_{2}, \ldots, n_{t}\right)$, where $n_{1} \geq n_{2} \geq \cdots \geq n_{t}$ be the unique short tree in which the $i$-th node at level 1 has $n_{i}$ children. For example, Figure 1 shows the tree $T_{5}(3,3,1,1,0)$. The number of nodes in the tree $T_{t}\left(n_{1}, n_{2}, \ldots, n_{t}\right)$ is $1+t+n_{1}+n_{2}+\cdots+n_{t}$. Every free tree of diameter at least 2 and at most 4 can be made into a short tree by taking a center to be the root.

Theorem 2.2 For every $\mathrm{t} \geq 2$ and $\mathrm{n} \geq 1$ there is a cyclic $\mathrm{T}_{\mathrm{t}}(\mathrm{n}, 1,0, \ldots, 0)$-code. 


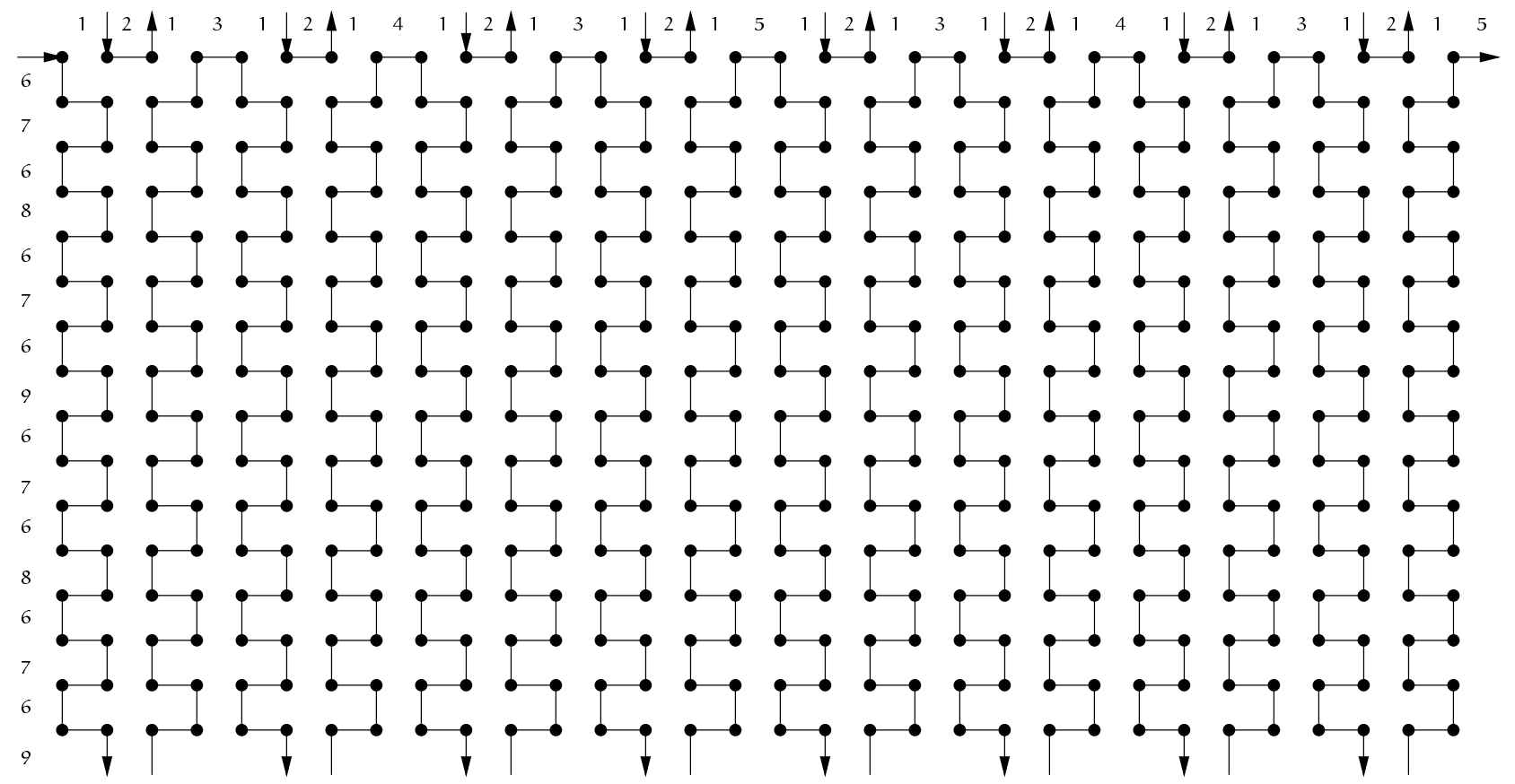

Figure 2: How to weave together the BRGC sequences $T_{4}$ and $T_{5}$ to get a $T_{4}(3,1,0,0)$-code.

Proof: We weave together a cyclic BRGC transition sequence over $\mathrm{Q}_{t}$ together with one over $\mathrm{Q}_{\mathfrak{n}+2}$. Thinking of $\mathrm{Q}_{\mathrm{t}+\mathfrak{n}+2}$ as $\mathrm{Q}_{\mathrm{t}} \times \mathrm{Q}_{\mathfrak{n}+2}$, one sees that the Hamilton cycles given by the BRGC for $t$ and $n+2$ induce a $2^{t}$ by $2^{n+2}$ toroidal grid structure in $Q_{t+n+2}$.

Assume that the nodes are labelled so that 1 is the root, that its children are $n+$ $3, \ldots, n+t+2$, that $n+t+2$ has only child 2 , and that the children of $n+3$ are $3,4, \ldots, n+2$. Label the rows of the grid with the cyclic transition sequence for the BRGC, using $n+3, \ldots, n+t+2$, and the columns with $1,2, \ldots, n+2$. On this toroidal spanning subgraph, we weave a specific Hamilton cycle which starts at the left upper point and follows the patterns illustrated in Figure 2, which uses $t=4$ and $n+2=5$. Beginning with a vertical move, we zigzag down the $2^{\mathrm{t}}$ rows, wrapping round in column 2 , then zigzag back up. This up-down zig-zag is repeated $2^{\mathfrak{n}}$ times and ends at the right upper point, which attaches to the left upper point to create the Hamilton cycle on $Q_{t+n+2}$.

In the columns, note that if a transition is not 1 (these transitions occur only in the first and last rows), then it is adjacent only to $n+3$ or $n+t+2$; furthermore, it is only 2 , which occurs $2^{\mathfrak{n}}$ times as a column, that is adjacent to $n+t+2$; the remaining vertices $3,4, \ldots, n+2$ are adjacent only to $n+3$.

Corollary 2.3 For any complete multipartite graph G, there is a cyclic G-code. 
THE ELECTRONIC JOURNAL OF COMBINATORICS 3 (1996), \#R11

Proof: Let the number of vertices in $G$ be $n$. Consider any 2 of the partite sets, $R$ and $P$, in $G$, where $|P|=m$ : If either set has only 1 vertex, then $G$ contains $S_{n}$ as a spanning tree and we can generate a BRGC from it. If $R$ and $P$ each contain 2 vertices, and $G$ is bipartite, then the sequence $1,2,3,2,1,2,3,4,3,2,1,2,3,2,1,4$, using vertices labelled sequentially on the 4-cycle, can be used.

Otherwise, choose vertex $r \in R$ and mark its edges that are incident with a vertex in $P$. Choose another vertex in $R$ and mark its edge to some specific vertex $s \in P$. Choose another vertex $\mathrm{q} \neq \mathrm{s} \in \mathrm{P}$ and mark its incident edges to every remaining unconsidered vertex in $R$ and the other partite sets. We can see that the marked edges of $G$ constitute a spanning $T_{m}(n-m-2,1,0, \ldots, 0)$ subgraph of $G$, with $r$ as the root. By Theorem 2.2, this spanning subgraph guarantees a cyclic G-code.

Theorem 2.4 For every tree $\mathrm{T}$ of diameter 4 , there is a cyclic $\mathrm{T}$-code.

Proof: Let $m=n_{1}+n_{2}+\cdots+n_{t}$ be the total number of leaves in $T=T_{t}\left(n_{1}, n_{2}, \ldots, n_{t}\right)$, and let $n=1+t+m$ be the total number of vertices. Consider the multipartite graph $G$ whose partite sets have sizes $n_{1}, n_{2}, \ldots, n_{t}$; label its vertices with the corresponding leaves of $T_{t}\left(n_{1}, n_{2}, \ldots, n_{t}\right)$. By Corollary 2.3, there is a cyclic G-transition sequence, $\Gamma=g_{1}, g_{2}, \ldots, g_{M}$, where $M=2^{m}$.

Now, consider $S_{t+1}$, with central vertex being the root of $T_{t}\left(n_{1}, n_{2}, \ldots, n_{t}\right)$ and the leaves being the children of the root: By Lemma 2.3, we can choose any 2 distinct leaves as a start-finish combination, to produce a $S_{t+1}$-transition sequence of length $2^{t+1}-1$. Choose such a sequence, $\Sigma_{i}$, for each $g_{i}, g_{i+1}$ pair in $\Gamma$, such that the starting vertex is adjacent to $g_{i}$ and the ending vertex is adjacent to $g_{i+1}$, using $T_{t}\left(n_{1}, n_{2}, \ldots, n_{t}\right)$ to determine adjacencies. Also, choose a sequence $\Sigma_{M}$ such that the starting vertex is adjacent to $g_{M}$ and the finish vertex is adjacent to $g_{1}$. Form a new sequence, $\Omega=g_{1}, \Sigma_{1}, g_{2}, \Sigma_{2}, \ldots, g_{M}, \Sigma_{M}$ of length $2^{m}\left(2^{\mathrm{t}+1}-1\right)+2^{\mathrm{m}}=2^{\mathrm{n}}$.

We show that $\Omega$ is the cyclic transition sequence for a cyclic $T_{t}\left(n_{1}, n_{2}, \ldots, n_{t}\right)$-code: Each $g_{i}$ bit change is matched with a Gray code corresponding to the $S_{t+1}$-bits, and the $\mathrm{g}_{i}$ 's themselves are a cyclic G-transition sequence. Essentially, we are tracing out a H-path in a $2^{\mathrm{m}}$ by $2^{\mathrm{t}+1}$ grid graph.

To show that the transition sequence is cyclic, we investigate the parity of all the vertices in the $\Sigma_{i}$ 's. Each $\Sigma_{i}$ has length $2^{t+1}-1$ and can be made cyclic by appending an $r$ to it, meaning it encounters only the central vertex (the original root) an odd number of times. But since there are an even number of $\Sigma_{i}$ 's, the total number of $r$ 's in $\Omega$ is even. Therefore, by Theorem 1.1, we know $\Omega$ is a cyclic transition sequence.

Theorem 2.5 For every tree $\mathrm{T}$ of diameter 3, there does not exist a cyclic $\mathrm{T}$-code. 
Proof: Given the diameter 3 tree $T=T_{t}(m, 0, \ldots, 0)$, let $r$ and $f$ be its centers, and let the leaves adjacent to $f$ be $w_{1}, w_{2}, \ldots, w_{m}$. Assume that $H=\mathbf{b}_{1}, \mathbf{b}_{2} \ldots, \mathbf{b}_{\mathrm{N}}$ is a cyclic $T$-code, whose cyclic transition sequence is $\tau(\mathrm{H})$; we will derive a contradiction.

Divide $\tau(H)$ into all the subsequences which begin with and contain exactly 1 occurence of any $w_{i}$; subsequences of three possible forms result: The A-type may exist and has the form $w_{i} f$. The B-type may exist and has the form $w_{i}$ frf. The C-type is necessary and has the form $w_{i} f r \cdots r f$.

Since $\tau(H)=t_{1} t_{2} \cdots t_{N}$ is cyclic we may assume that it begins with a subsequence that is a C-type. Let $k$ be the index of the last $f$ in this subsequence. Let $x^{\prime}=O^{n} t_{1} t_{2} \cdots t_{k-2}$, $\mathrm{x}=\mathrm{x}^{\prime} \mathrm{t}_{\mathrm{k}-1}=\mathrm{x}^{\prime} \mathrm{r}, \mathrm{y}=\mathrm{xt}_{\mathrm{k}}=\mathrm{xf}$, and $\mathbf{y}^{\prime}=\mathbf{y r}=\mathrm{x}^{\prime} \mathrm{f}$.

There must be a unique index $\mathbf{j}$ for which $\mathbf{y}^{\prime}=\mathbf{b}_{j}$. Denote $\mathbf{u}=\mathbf{b}_{j-1}$ and $\mathbf{v}=\mathbf{b}_{j+1}$. There are four mutually exclusive cases to consider since either $r$ or a $w_{i}$, but not both, must delimit $\mathbf{y}^{\prime}$, either on the right or on the left. These cases are (a) $\tau\left(\mathbf{u}, \mathbf{y}^{\prime}\right)=r$, (b) $\tau\left(\mathbf{y}^{\prime}, \mathbf{v}\right)=$ $r$, (c) $\tau\left(\mathbf{u}, \mathbf{y}^{\prime}\right)=w_{i}$, and (d) $\tau\left(\mathbf{y}^{\prime}, \mathbf{v}\right)=w_{i}$. In case (a), we must have $\mathbf{u}=\mathbf{y}^{\prime} \mathbf{r}=\mathbf{y}$, and in case (b), we must have $\mathbf{v}=\mathbf{y}^{\prime} \mathbf{r}=\mathbf{y}$. But both these cases cannot occur as $\mathbf{y}$ by definition is delimited by $f$ and $w_{i}$, and not by $r$. In case (c), we must have $\mathbf{b}_{j+1}=\mathbf{y}^{\prime} f=x^{\prime}$ which means that the subsequence, $w_{i}$ frf is contained within this subsequence. But by definition, a C-type subsequence cannot contain a B-type subsequence. In case (d), we conclude that $\mathbf{b}_{j-1}=\mathbf{y}^{\prime} f=\mathbf{x}^{\prime}$. This means that $\mathbf{x}^{\prime}$ is delimited on the right by $f$, which is not true.

Conjecture 2.1 If $\mathrm{T}$ is a tree then there is a cyclic $\mathrm{T}$-code if and only if the diameter of $\mathrm{T}$ is 2 or 4 .

If the conjecture is true, then for $n \geq 6, P_{n}$-codes do not exist, where $P_{n}$ is a path of length $n$. A natural way to add edges is to take the square of the graph.

Question 2.2 Does a $\mathrm{P}_{\mathrm{n}}^{2}$-code exist for all $\mathrm{n} \geq 1$ ?

The question asked in the introduction about Gray codes being lifted to Hamilton cycles on the cube-connected cycle is equivalent to the following question.

Question 2.3 Does a cyclic $\mathrm{C}_{n}$-code for $\mathrm{n} \geq 5$ exist?

\section{$2.1 \quad$ Experimental results}

We have obtained a complete classification of all G-codes for graphs on at most 6 vertices, and for some graphs on 7 vertices. Refer to Figure 3. Any graph $\mathrm{G}$ of order at most six not illustrated contains, as a spanning subgraph, one or more of these graphs having a cyclic Gray Code, and thus itself possesses a cyclic G-code. For example, any 6 vertex connected graph with 6 edges, which is not a 6-cycle, must contain (6.2) or (6.3) as a spanning subgraph. 
Graphs of Size 3

1)

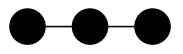

Graphs of Size 4

1)

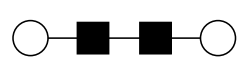

2)

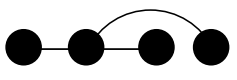

3)

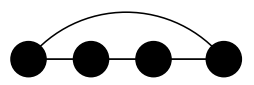

Graphs of Size 5

a)

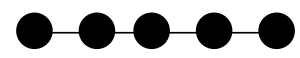

2)

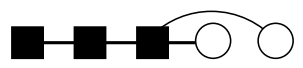

3)

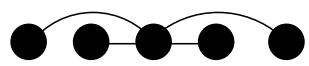

Graphs of Size 6

1) $\square-O-O$

2)

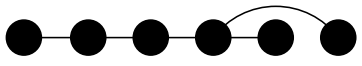

3)

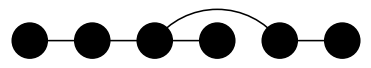

4)

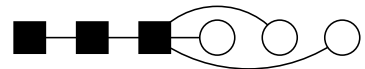

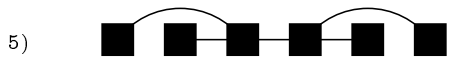

6)

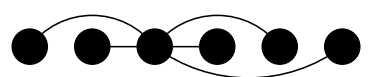

7)

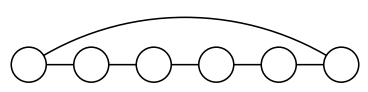

KEY

no transition sequence possible

transition sequence possible

cyclic transition sequence possible

Figure 3: Minimal subgraphs of order 3 to 6 . Every connected graph contains one of these as a spanning subgraph. 
THE ELECTRONIC JOURNAL OF COMBINATORICS 3 (1996), \#R11

\section{Directed G-codes}

Let $\mathrm{G}$ be a directed graph. We make a make here a few small observations about directed G-codes All the definitions in the Introduction carry over to directed G-codes.

Lemma 3.1 If there is a cyclic G-code, then $\mathrm{G}$ is strongly connected.

Proof: Choose any 2 vertices, $u$ and $v$ in $G$. Let $H=\mathbf{b}_{1}, \mathbf{b}_{2}, \ldots, \mathbf{b}_{N}$ be a cyclic G-code such that $u=\tau\left(\mathbf{b}_{1}, \mathbf{b}_{2}\right)$. Since the bit in position $v$ must change, there must be a path from $u$ to $v$ in the graph of transitions, which is a subgraph of $G$.

That a strongly connected graph is not sufficient to produce a G-code is demonstrated by considering any directed cycle of $n$ vertices. This graph forces the transition sequence to repeat the sequence after $2 n$ which is less than $2^{n}$ for $n \geq 3$.

Question 3.1 Does a cyclic transition sequence require that its graph of transitions have at least one bi-directional edge?

\section{Acknowledgement}

We thank Stephen Chen of the Department of Computer Science of Griffiths University, Australia, for motivating this paper by posting the essence of Question 2.3 to the newsgroup sci.math.research. We also thank Luis Goddyn, Wendy Myrvold, and Glenn Hurlbert for helpful comments on an earlier draft of this paper.

\section{References}

[1] J. Bitner, G. Ehrlich, and E. Reingold, "Efficient Generation of the Binary Reflected Gray Code and its Applications," Communications of the ACM, 19 (1976) 517-521.

[2] E.N. Gilbert, Gray Codes and paths on the n-cube, Bell Systems Technical Journal, 37 (1958) 815-826.

[3] L. Goddyn, G.M. Lawrence, and E. Nemeth, Gray Codes with optimized run lengths, Utilitas Mathematica, 34 (1988) 179-192.

[4] F. Thomson Leighton, Introduction to Parallel Algorithms and Architectures: Arrays, Trees, Hypercubes, Morgan Kaufmann, 1992.

[5] A. Nijenhuis and H. S. Wilf, Combinatorial Algorithms, Second Edition, Academic Press, 1978. 
THE ELECTRONIC JOURNAL OF COMBINATORICS 3 (1996), \#R11

[6] Reingold, Nievergelt, and Deo, Combinatorial Algorithms: Theory and Practice, Prentice Hall, 1977.

[7] Carla D. Savage and Peter Winkler, Monotone Gray Codes and the Middle Levels Problems, J. Combinatorial Theory, Series A, 70 (1995) 230-248.

[8] H.S. Wilf, Combinatorial Algorithms: An Update, SIAM, CBMS 55. 